\title{
Construcción y Validación de la Escala de Consumo Patológico para adolescentes de Lima Metropolitana, 2013
}

\author{
Construction and validation of the Scale of Consumerist Pathological for adolescents of metropolitan \\ Lima, 2013
}

Luzmila Abigail García García', Jakeline Peralta Casanova² ${ }^{2}$ Renzo Felipe Carranza Esteban ${ }^{3}$

\begin{abstract}
RESUMEN
Objetivo: Construir y determinar las propiedades psicométricas de la Escala de Consumo Patológico, para adolescentes de Lima Metropolitana. Material y Métodos: La metodología utilizada fue de diseño no experimental, de tipo de psicométrico. El instrumento consta de 32 ítems y se compone de dos dimensiones: 1) consumo por compulsión (16 ítems) y 2) consumo por evasión (16 ítems). Diseñado para ser utilizado en adolescentes entre 15 y 19 años de edad, valorando de forma cualitativa y cuantitativa la gravedad de los síntomas. Los participantes se escogieron mediante un muestreo no probabilístico de tipo por conveniencia, de ambos sexos. Resultados: La validez de contenido del instrumento fue ejecutado mediante el criterio de jueces; aplicándose el coeficiente $\mathrm{V}$ de Aiken; alcanzándose niveles adecuados de significación estadística en cuanto a claridad, sencillez y precisión de reactivos. En relación a la validez de constructo, el KMO presentó un valor de 0.870 indicando la posibilidad para el uso del análisis factorial proyectándose, como resultados, un nivel de significancia alto; al efectuarse el análisis por componentes rotados de Varimax, se obtuvo como resultado dos factores que muestran adecuadas correlaciones debido a la saturación de los ítems. Sin embargo, la varianza, explicada por dos factores, presentó un valor de $35.124 \%$. Al respecto el nivel de consistencia interna, de la escala en general, alcanzó un alfa de Cronbach de (0.791), evidenciando significancia, así como en ambas dimensiones: 1) dimensión consumo por compulsión, Alfa de Cronbach (0.844) y 2) dimensión de consumo por evasión, Alfa de Cronbach (0.879); además, al correlacionar los ítems con el test, se evidenció una significancia menor a $(\mathrm{p}<0.05)$, evidenciando la validez de los factores propuestos. Conclusiones: Finalmente se concluye que la Escala de Consumo Patológico es válida, fiable y apta para su uso, ya que se ajusta a las propiedades psicométricas requeridas.
\end{abstract}

Palabras clave: Consumo patológico, consumo por compulsión, consumo por evasión, confiabilidad, validez.

\begin{abstract}
Objective: To construct and determine the psychometric properties of the scale Pathological consumption for adolescents in metropolitan Lima. Material and Methods: The methodology used was non-experimental design, psychometric type. The 32-item instrument coast and consists of two dimensions: 1) compulsive consumption (16 items) and 2) use evasion (16 items). Designed for use in adolescents 15 to 19 years old, assessing qualitatively and quantitatively the severity of symptoms. Participants were selected using a non-probability convenience sampling rate of both sexes. Results: The content validity of the instrument was executed by the criterion validity of judges; multiplied by the coefficient V of Aiken; reaching adequate levels of statistical significance for clarity, simplicity and accuracy of reagents. Regarding construct validity, the KMO value of 0.870 submitted indicating the possibility for the use of factor analysis, projecting as a result, a high level of significance; Upon analysis Varimax rotated components, is obtained as the result of two factors show correlations appropriate due to saturation of the items. However, the variance explained by two factors, provided a value of $35.124 \%$. In this regard the level of internal consistency of the scale in general reached a Cronbach's alpha (0.791), showing significance, as well as in two dimensions: 1) dimension compulsive consumption, Cronbach's alpha (0.844) and 2) consumer aspects evasion, Cronbach's alpha (0.879), correlating well with the test items, is to lower $(\mathrm{p}<0.05)$ showed significance, demonstrating validity of the proposed factors. Conclusions: The paper concludes that the consumption Pathological scale is valid and reliable, suitable for use as it fits the psychometric properties required.
\end{abstract}

Keywords: Pathological consumption, compulsive consumption, consumption evasion, reliability, validity.

${ }^{1}$ Psicóloga del Colegio Adventista Las Américas, Tumbes

${ }^{2}$ Psicologa del programa BECA 18, Universidad Peruana Unión, Lima

${ }^{3}$ Profesor asociado, director de Investigación de la E.A.P de Psicología; Facultad de Ciencias de la Salud, Universidad Peruana Unión, Lima

Correspondencia: luzmbi_23@hotmail.com 


\section{INTRODUCCIÓN}

El consumo es una experiencia normal y rutinaria, sin embargo, en la actualidad ha pasado de ser una forma de satisfacer las necesidades básicas a un estilo de vida, tergiversando, como dice Delgado (2010), el sentido de identidad de la sociedad, generándose de esta manera un sentido de dependencia psicológica, que da paso al consumo patológico, siendo su diagnóstico complejo.

Por su parte, esta conducta repetitiva se ha convertido en un canal para satisfacer necesidades emocionales, generando vulnerabilidad y pérdida de control de sí mismo (Moreschi, 2006). A su vez esta patología no es reconocida en los manuales de diagnóstico como el CIE 10 o DSM IV, probablemente debido a que es un fenómeno de la edad moderna, en las que solo existen criterios para adicciones a los juegos patológicos o dependencia a sustancias (Choliz, 2013), además hace comorbilidad con otras patologías del estado del ánimo, del control de impulsos, trastorno obsesivo compulsivo, entre otros" (Cala et al. 2011, p. 72).

Por otro lado, se evidencian datos estadísticos que explican el nivel creciente de consumo tal como afirma Santamarta (2007), al mencionar que existen 1.700 millones de consumidores y que la sociedad de consumo, a nivel mundial, está integrada por 1.728 millones de personas; siendo los países occidentales los que consumen más; sin irse muy lejos, en Lima Metropolitana se toma en cuenta un $47 \%$ de hogares que tienen un miembro pagando algún tipo de deuda. Además, las ventas de los supermercados bordearon los 10,000 millones de nuevos soles durante el 2013, lo que representa un crecimiento de $10 \%$ respecto al 2012, según el banco Scotiabank. (diario Gestión, 2 de junio del 2013).

Otro factor que se considera importante es que, en el contexto peruano y específicamente en Lima Metropolitana, la población adolescente representa un nivel de riesgo elevado orientado hacia la adquisición de diferentes patologías, entre ellas el consumo patológico (Rodríguez, 2001).

Sin embargo, después de visualizar el problema sobre el consumo patológico y sus consecuencias, como también su complejo diagnóstico, se detecta que la realidad peruana no cuenta con instrumentos que evalúen esta problemática. Por ello, la contribución de este trabajo radica en el diseño y construcción de una escala para medir el consumo patológico que sea válido y fiable; y a su vez distinguir el mecanismo de su presentación, es decir, si el consumo patológico está orientado a una compulsión o a un consumo patológico por evasión, y motivar su la utilización en diferentes áreas de la psicología.

\section{MATERIAL Y MÉTODOS}

La metodología utilizada fue de diseño no experimental, porque no hubo manipulación de variables, de corte transversal ya que se efectuó en un tiempo determinado y de tipo psicométrico, porque busca determinar las propiedades psicométricas de validez de contenido, la validez de constructo y nivel de fiabilidad de la escala de consumo patológico, según la realidad social y cultural de los adolescentes de Lima Metropolitana (Fernández y Batista, 1967).

Los datos utilizados, en este estudio, se obtuvieron a partir de un muestreo no probabilístico de tipo por conveniencia; los participantes fueron 400 adolescentes del cuarto y quinto año de secundaria y nivel preuniversitario de Lima Metropolitana, distribuido como sigue: 1 academia preuniversitaria, 4 colegios particulares y 1 instituto tecnológico (delimitando la muestra).

Se seleccionaron personas entre 15 y 19 años porque son la población meta del instrumento, de ambos sexos con una edad media de 3 (DT= 0 ). Siendo que 80 protocolos no se contestaron adecuadamente, omitiéndose respuestas, quedando un total de 320 personas, donde el $54.1 \%$ fue de sexo femenino y el $45.9 \%$ de sexo masculino, asimismo, hubo un $90.06 \%$ de jóvenes solteros y un $0.93 \%$ de casados. Finalmente, de todos los jóvenes que conformaron la muestra, un $72.2 \%$ depende de sus padres económicamente, un $18.1 \%$ vive solo, pero sus padres le envían dinero y un $9.7 \%$ se autosostiene. $\mathrm{La}$ recogida de datos se dio entre los meses de noviembre y diciembre del 2013.

Entendiendo el consumo patológico, como la adquisición de todo tipo de bienes o servicios de manera exagerada que produce un estado de ansiedad y compulsión continua, en el que la persona nunca está satisfecha y necesita consumir constantemente; basando su identidad en lo que obtiene, afectando directamente su salud mental y emocional, su funcionalidad a nivel laboral, social, familiar y económico; presentado en dos mecanismos de consumo: por compulsión y por evasión. En base a esta definición conceptual, la prueba fue desarrollada como un constructo bidimensional; dimensión consumo por compulsión y la dimensión consumo por evasión, los cuales están conformados por 8 indicadores, constituidos a su vez por 32 ítems. 
Tabla 1

Operacionalización del constructo, consumo patológico

\begin{tabular}{|c|c|c|c|}
\hline Variable & Dimensión & Definición & Indicadores \\
\hline \multirow[t]{2}{*}{$\begin{array}{l}\text { Consumo } \\
\text { patológico }\end{array}$} & $\begin{array}{l}\text { Consumo por } \\
\text { compulsión }\end{array}$ & $\begin{array}{l}\text { Tipo de conducta de consumo } \\
\text { excesivo, molesto para la vida de los } \\
\text { individuos. }\end{array}$ & $\begin{array}{l}\text { Sensación de tensión } \\
\text { creciente }\end{array}$ \\
\hline & Consumo por evasión & $\begin{array}{l}\text { Respuesta incontrolable para obtener, } \\
\text { usar o experimentar un sentimiento, } \\
\text { que conduce al individuo a implicarse } \\
\text { de modo repetitivo en una conducta } \\
\text { nociva, entrando en un estado de } \\
\text { compulsión después de haber sido } \\
\text { condicionado y reforzar su conducta } \\
\text { tras la repetición (Faber, 0'Guinn y } \\
\text { Krych, } 1987 \text { y Bauman, 2007). } \\
\text { Conducta de consumo basado en } \\
\text { motivaciones o esquemas cognitivos. } \\
\text { Debido a tres factores: 1) sentido de } \\
\text { búsqueda para suplir un sentimiento } \\
\text { de malestar y bajo estado de ánimo. } \\
\text { 2) deseo de llamar la atención y } \\
\text { buscar resaltar en su entorno y } 3 \text { ) } \\
\text { construcción y expresión del propio } \\
\text { yo, debido a un sentimiento de vacío } \\
\text { existencial; todo ello con el fin de } \\
\text { lograr un sentimiento de bienestar } \\
\text { (Aravena et al. 2006) }\end{array}$ & $\begin{array}{ll}\text { - } & \text { Pensamiento } \\
\text { - } & \text { irracional. } \\
\text { - } & \text { Sentimiento autocontrol. } \\
& \text { bienestar. } \\
\text { - } & \text { Compensadores del } \\
\text { estado de ánimo. } \\
\text { - } \quad \text { Consumir para } \\
\text { llamar la atención. } \\
\text { - } \quad \text { Sentimientos de } \\
\text { bienestar. } \\
\text { - Construcción de } \\
\text { identidad. }\end{array}$ \\
\hline
\end{tabular}

\section{Instrumentos}

Escala de consumo patológico (ECP).

Es una escala de 32 ítems, 16 ítems en la dimensión consumo por compulsión y 16 ítems en la dimensión consumo por evasión, con tres alternativas de respuestas graduadas (casi nunca, a veces y a menudo), sustentado en el marco teórico del constructo, siendo que es muy complejo dar a conocer una cantidad tope de consumo. La población de la escala se centra en individuos entre 15 y 19 años de edad, de ambos sexos; su objetivo es evaluar el

\section{Tabla 2}

Tipo de respuestas de la Escala de Consumo Patológico nivel de consumo patológico y distinguirlo según sus mecanismos (compulsión y evasión) en adolescentes.

Este instrumento consiste en una hoja con las indicaciones pertinentes para poder desarrollarlo, tomando en cuenta las alternativas y el puntaje que estas equivalen, siendo el mayor puntaje 3 , se debe considerar que la sumatoria final indicará que a mayor porcentaje será mayor consumo y viceversa. La forma de respuesta es marcar con un aspa la que más se adecúa a su caso, como se describe en la tabla 2.

\begin{tabular}{ll}
\hline Respuestas & Puntajes \\
\hline Casi nunca & 1 \\
A veces & 2 \\
A menudo & 3 \\
\hline
\end{tabular}

La calificación de este instrumento se basa en la sumatoria final de cada respuesta, siendo que cada puntaje indicará, según los baremos, el rango o nivel donde se ubica el grado de consumo del individuo el cual puede ser leve, moderado y severo considerando el sexo como se muestra en la tabla 3; ya que, de acuerdo al marco teórico que sustenta este trabajo, se evidencia un mayor consumo en mujeres que en varones. 
Tabla 3

Clasificación de categorías para la interpretación diagnóstica según el sexo

\begin{tabular}{lll}
\hline Categoría & Puntaje & \\
\hline & Mujeres & Hombres \\
\cline { 2 - 3 } Normal & $36-44$ & $34-45$ \\
Leve & $46-51$ & $46-50$ \\
Moderado & $52-55$ & $51-56$ \\
Severo & $56-68$ & $57-73$ \\
\hline
\end{tabular}

\section{Procedimiento}

La construcción de esta prueba se basó en el modelo cognitivo conductual, el cual se respalda en diferentes pilares teóricos que orientan a una clara explicación de la variable de estudio, siendo estos: el condicionamiento clásico, condicionamiento operante, teoría del aprendizaje social y los modelos cognitivos de Beck y Ellis. De esta manera, por medio del condicionamiento clásico se explica la adquisición de una conducta de consumo, la cual es reforzada por medio del condicionamiento operante; además se asume que la conducta de consumo es aprendida, ya que existe una participación activa de las personas y el ambiente para modelar el comportamiento, finalmente se hace hincapié en la existencia de pensamientos, sentimientos, creencias, expectativas y conocimientos previos distorsionados en relación al consumo, que formarán esquemas cognitivos (creencias básicas) relativamente estables y que determinarían la conducta.

Por lo que se construyeron ítems para medir el consumo patológico manifestado en dos mecanismos, un consumo patológico por compulsión o por evasión, los que fueron sometidos a criterio de jueces que evaluaron tanto el ajuste al modelo conceptual como la redacción y claridad de las afirmaciones. Los jueces fueron: cinco psicólogos clínicos, todos ellos académicos universitarios, que revisaron de manera independiente los ítems propuestos. A partir de sus observaciones, se conservaron todos los ítems, sin embargo, se reformularon los ítems 29 y 31 . Una vez concluida esta fase, se consiguió la autorización de la directora de la E.A.P. de Psicología y de Investigación para la aplicación a la muestra piloto.
El instrumento se aplicó a estudiantes de cuarto y quinto año de secundaria de diferentes colegios, así como de institutos o academias preuniversitarias, haciendo un total de 400 personas, sin embargo, ochenta de ellos no contestaron algunos ítems de los cuestionarios y fueron eliminados del estudio, quedando 320 participantes en la muestra final y utilizando aproximadamente 20 minutos para el desarrollo de la prueba. A los estudiantes se les explicó, en términos generales, el objetivo del estudio y se les solicitó su colaboración voluntaria, informándoles sobre el carácter confidencial de los datos recogidos $\mathrm{y}$, según el caso, firmando un documento de consentimiento informado. Si algún estudiante no quería participar, no se le entregaba el instrumento. De esta manera, se cumplió con las normas éticas establecidas para este tipo de estudio.

A partir de los datos obtenidos, se realizó un análisis estadístico, utilizando el paquete de datos SPSS recurriendo, en primer lugar, al análisis de fiabilidad total de la escala y de sus dimensiones, calculando el índice de la consistencia interna mediante el coeficiente Alfa de Crombach, el cual es muy sensible al tamaño de la muestra y la cantidad de reactivos o ítems (a menor cantidad de ítems más bajo el valor alfa y viceversa a mayor cantidad de ítems más alto valor alfa); y $\mathrm{V}$ de Aiken (validez por jueces), así como análisis de correlación y el análisis factorial (validez factorial), respectivamente. Luego se recurrió a estadísticos descriptivos de posición para presentar la baremación del test. 


\section{RESULTADOS}

\section{Confiabilidad}

Fiabilidad mediante la técnica de Alfa de Cronbach de la escala total.

Tabla 4

Estimaciones de fiabilidad mediante el coeficiente de Alfa

\begin{tabular}{lll}
\hline Dimensiones & $N^{\circ}$ de ítems & Alfa de Cronbach \\
\hline Consumo por compulsión & 16 & .844 \\
Consumo por evasión & 16 & .879 \\
Escala total & 32 & .791 \\
\hline
\end{tabular}

La tabla 4 presenta las estimaciones de la fiabilidad (consistencia interna), obtenidos a partir de una muestra de 320 adolescentes de Lima Metropolitana. Los índices del Alpha Cronbach estimados para cada dimensión del inventario son elevados, porque superan los valores de punto de corte de 0.70. En conclusión, la escala de consumo patológico presenta alta consistencia interna.

Fiabilidad mediante la técnica de Alfa de Cronbach del factor consumo por compulsión y consumo por evasión.

Tabla 5

Índices de consistencia interna mediante el Alfa de Cronbach de la dimensión consumo por compulsión.

\begin{tabular}{lll}
\hline Ítem & Correlación elemento-total corregida & Valor alfa si el ítem es eliminado \\
\hline P1 & .403 & .838 \\
P2 & .526 & .832 \\
P3 & .521 & .832 \\
P4 & .379 & .839 \\
P5 & .421 & .837 \\
P6 & .335 & .842 \\
P7 & .515 & .832 \\
P8 & .546 & .830 \\
P10 & .380 & .841 \\
P11 & .577 & .829 \\
P12 & .361 & .840 \\
P13 & .293 & .845 \\
P14 & .402 & .838 \\
P15 & .489 & .834 \\
P16 & .668 & .825 \\
\hline
\end{tabular}

La tabla 5 muestra que los ítems tienen una adecuada contribución a la dimensión consumo por compulsión, también se puede observar que el valor alfa del ítem 13 presenta un ligero aumento, en contraste con el valor alfa de la dimensión respectiva; sin embargo, se decide mantener este ítem en este estudio, dado las circunstancias del caso y se recomienda su eliminación en estudios posteriores. 
Tabla 6

Índices de consistencia interna mediante el Alfa de Cronbach de la dimensión consumo por evasión.

\begin{tabular}{ccc}
\hline Ítem & Correlación elemento-total corregida & Valor Alfa si ítem es eliminado \\
\hline P17 & .504 & .873 \\
P18 & .394 & .877 \\
P19 & .458 & .875 \\
P20 & .325 & .880 \\
P21 & .362 & .878 \\
P22 & .399 & .877 \\
P23 & .599 & .869 \\
P24 & .599 & .869 \\
P25 & .457 & .875 \\
P26 & .605 & .869 \\
P27 & .595 & .870 \\
P28 & .603 & .869 \\
P29 & .650 & .866 \\
P30 & .460 & .875 \\
P31 & .709 & .863 \\
P32 & .617 & .868 \\
\hline
\end{tabular}

La tabla 6 muestra que cada uno de los ítems logra una buena contribución a la dimensión consumo por evasión, indicando que dicha dimensión presenta una alta consistencia interna, no obstante se evidencia que el valor alfa obtenido por el ítem 20, ligeramente alto según el alfa total de la dimensión respectiva, sugiere su eliminación, sin embargo, se decide mantener este ítem, debido a que se sustenta en el marcoteórico propuesto, el cual afirma que el consumo por evasión también se encuentra asociado a estados de manía (Black, 2010).

\section{Validez}

Validez de contenido mediante el criterio de jueces.

Inicialmente la validez de contenido del instrumento fue estudiado mediante la validez de criterio de jueces, aplicándose el coeficiente V de Aiken (Escurra, 1988). Se entregó el instrumento, con las correspondientes definiciones operacionales, (tanto para la escala total como para cada uno de los ítems), a un grupo de 5 jueces que independientemente evaluaron si la correspondencia de los ítems al universo de contenido es precisa, clara y sencilla, llegando a determinarse la reformulación de los ítems 29 y 31 .

Tabla 7

Coeficiente V de Aiken para validez de contenido de la escala.

\begin{tabular}{lll}
\hline Test & V Aiken & P \\
\hline Adecuadas características, forma de aplicación y estructura de la escala & $1^{* *}$ & 0.001 \\
Orden adecuado de las preguntas de la escala & $1^{* *}$ & 0.001 \\
¿Existe dificultad para entender las preguntas de la escala? & $1^{* *}$ & 0.001 \\
¿Existen palabras difíciles de entender en los ítems 0 reactivos de la escala? & $1^{* *}$ & 0.001 \\
¿Las opciones de respuesta están suficientemente graduadas y pertinentes para cada & $1^{* *}$ & 0.001 \\
ítem 0 reactivo de la escala? & \\
¿Los ítems o reactivos del instrumento tienen correspondencia con la dimensión a la que & $1^{* *}$ & 0.001 \\
pertenecen en el constructo? & & \\
\hline${ }^{* *} p<0.01$ & & \\
Jueces $=5$ & &
\end{tabular}


La tabla 7 presenta resultados que indican un nivel de claridad y sencillez del instrumento, según la consistencia de los jueces en sus apreciaciones concernientes a la forma global de la escala, después de la reformulación de los ítems 29 y 31. Lo que significa que la escala de consumo patológico presenta validez de contenido, bajo este tipo de criterio.

\section{Validez de constructo mediante la técnica de análisis ítems test}

\section{Tabla 8}

Correlación item - subtest

\begin{tabular}{|c|c|c|}
\hline $\begin{array}{r}\text { Dimensión: consumo } \\
\text { Ítem }\end{array}$ & $r$ & $p$ \\
\hline P1 & $.492\left({ }^{* \star}\right)$ & .000 \\
\hline P2 & $.600\left(^{* \star}\right)$ & .000 \\
\hline P3 & $.605\left(^{\star \star}\right)$ & .000 \\
\hline P4 & $.458\left(^{\star \star}\right)$ & .000 \\
\hline P5 & $.503\left({ }^{\star \star}\right)$ & .000 \\
\hline P6 & $.431\left(^{\star \star}\right)$ & .000 \\
\hline P7 & $.604\left({ }^{* \star}\right)$ & .000 \\
\hline P8 & $.634\left({ }^{\star *}\right)$ & .000 \\
\hline P9 & $.644\left({ }^{\star \star}\right)$ & .000 \\
\hline P10 & $.492\left({ }^{\star \star}\right)$ & .000 \\
\hline P11 & $.652\left({ }^{\star \star}\right)$ & .000 \\
\hline P12 & $.448\left({ }^{\star *}\right)$ & .000 \\
\hline P13 & $.398\left({ }^{* \star}\right)$ & .000 \\
\hline P14 & $.483\left({ }^{\star *}\right)$ & .000 \\
\hline P15 & $.578\left(^{* *}\right)$ & .000 \\
\hline P16 & $.725\left({ }^{\star \star}\right)$ & .000 \\
\hline
\end{tabular}

En las tablas 8 y 9 los cálculos estimados respecto a la correlación de los ítems a sus respectivas dimensiones, tomando como criterio de aceptación, correlaciones con significancia al $\mathrm{p}<0.05$, se observa que todos los coeficientes de correlación son significativos, evidenciando validez de los factores consumo por compulsión y consumo por evasión de la escala de consumo patológico.

La tabla 8 proyecta datos de correlación ítem subtest de la dimensión consumo por compulsión. 
Tabla 9

Correlación ítem-subtest

\begin{tabular}{|c|c|c|}
\hline $\begin{array}{l}\text { Dime } \\
\text { Ítem }\end{array}$ & $r$ & $p$ \\
\hline P1 & $.572\left(^{\star \star}\right)$ & .000 \\
\hline P2 & $\left..469^{* \star}\right)$ & .000 \\
\hline P3 & $.535\left(^{* \star}\right)$ & .000 \\
\hline P4 & $.418\left(^{* *}\right)$ & .000 \\
\hline P5 & $.439\left(^{* *}\right)$ & .000 \\
\hline P6 & $.485\left(^{* *}\right)$ & .000 \\
\hline P7 & $.669\left(^{* *}\right)$ & .000 \\
\hline P8 & $.673\left(^{* *}\right)$ & .000 \\
\hline P9 & $.539\left(^{\star \star}\right)$ & .000 \\
\hline P10 & $.579\left(^{\star \star}\right)$ & .000 \\
\hline P11 & $.653\left(^{* *}\right)$ & .000 \\
\hline P12 & $.666\left(^{\star \star}\right)$ & .000 \\
\hline P13 & $.713\left(^{\star *}\right)$ & .000 \\
\hline P14 & $.536\left(^{\star \star}\right)$ & .000 \\
\hline P15 & $.765\left(^{\star \star}\right)$ & .000 \\
\hline P16 & $.678\left(^{* \star}\right)$ & .000 \\
\hline
\end{tabular}

Validez de constructo mediante la técnica de análisis factorial

Tabla 10

KMO y prueba de Bartlett para evaluar la pertinencia del modelo factorial

\begin{tabular}{lll}
\hline $\begin{array}{l}\text { Medida de adecuación muestral de } \\
\text { Kaiser-Meyer-Olkin. }\end{array}$ & .870 \\
\hline $\begin{array}{l}\text { Prueba de esfericidad de } \\
\text { Bartlett }\end{array}$ & Chi-cuadrado aproximado & 3547.314 \\
& Gl & .496 \\
& Sig. & 0.000 \\
Varianza total explicada & & 35.124 \\
\hline
\end{tabular}

Se realizaron comprobaciones necesarias que avalen al análisis factorial como una técnica apta; utilizándose la medida de adecuación muestral de Kaiser-Meyer-Olkin (KMO).
Consecuentemente, en la Tabla 10 se observa que el valor de la estadística KMO (0.870) es alto. De modo que puede considerarse al análisis factorial como una técnica apropiada para llevarse a cabo en este estudio. 
Tabla 11.

Componentes rotados

\begin{tabular}{|c|c|c|}
\hline & Componente & \\
\hline Ítems & Consumo por compulsión & Consumo por evasión \\
\hline P1 & .479 & \\
\hline P2 & .633 & \\
\hline P3 & .617 & \\
\hline P4 & .492 & \\
\hline P5 & .535 & \\
\hline P6 & .400 & \\
\hline P7 & .589 & \\
\hline P8 & .587 & \\
\hline P9 & .622 & \\
\hline P10 & .438 & \\
\hline P11 & .690 & \\
\hline P12 & .489 & \\
\hline P13 & .346 & \\
\hline P14 & .483 & \\
\hline P15 & .568 & \\
\hline P16 & .717 & \\
\hline P17 & & .571 \\
\hline P18 & & .482 \\
\hline P19 & & .545 \\
\hline P20 & & .348 \\
\hline P21 & & .410 \\
\hline P22 & & .445 \\
\hline P23 & & .674 \\
\hline P24 & & .675 \\
\hline P25 & & .486 \\
\hline P26 & & .654 \\
\hline P27 & & .691 \\
\hline P28 & & .705 \\
\hline P29 & & .715 \\
\hline P30 & & .509 \\
\hline P31 & & .788 \\
\hline P32 & & .657 \\
\hline
\end{tabular}

Por otro lado, la extracción de dos factores es explicado por el $35.12 \%$ de la varianza, valor menor a lo sugerido (40\%). Sin embargo, se observa en la tabla 11 la saturación de los ítems en cada uno de las 2 dimensiones propuestas. Los pesos factoriales son en su totalidad mayores a 0.35 , esto significa que son suficientemente elevados y que estarían midiendo adecuadamente el constructo. Además se puede explicar dichos resultados al contar la escala de consumo patológico con solo dos dimensiones, siendo probable que el nivel de explicación aumente si se agregan más dimensiones. 


\section{Establecimiento de normas de interpretación o baremos}

Tabla 12

Baremos para mujeres

\begin{tabular}{|c|c|c|c|}
\hline PC & Consumo por compulsión & Consumo por evasión & Escala total \\
\hline 1 & 16.00 & 16.00 & 36.00 \\
\hline 2 & 16.60 & 16.00 & 36.60 \\
\hline 3 & 17.40 & 16.40 & 37.00 \\
\hline 4 & 18.00 & 18.00 & 37.20 \\
\hline 5 & 18.00 & 18.00 & 38.00 \\
\hline 10 & 18.00 & 18.00 & 40.00 \\
\hline 15 & 19.00 & 20.00 & 41.00 \\
\hline 20 & 19.00 & 21.00 & 43.00 \\
\hline 25 & 19.00 & 21.00 & 44.00 \\
\hline 30 & 20.00 & 22.00 & 46.00 \\
\hline 35 & 20.00 & 23.00 & 48.00 \\
\hline 40 & 21.00 & 25.00 & 49.00 \\
\hline 45 & 21.00 & 26.00 & 50.00 \\
\hline 50 & 22.00 & 27.00 & 51.00 \\
\hline 55 & 23.00 & 28.00 & 52.00 \\
\hline 60 & 24.00 & 29.00 & 53.00 \\
\hline 65 & 24.00 & 30.00 & 53.00 \\
\hline 70 & 25.00 & 31.00 & 54.00 \\
\hline 75 & 26.00 & 32.00 & 55.00 \\
\hline 80 & 27.00 & 33.00 & 56.00 \\
\hline 85 & 29.00 & 35.00 & 57.00 \\
\hline 90 & 31.00 & 36.00 & 58.00 \\
\hline 95 & 33.00 & 37.00 & 62.00 \\
\hline 96 & 33.80 & 38.00 & 63.00 \\
\hline 97 & 34.00 & 38.00 & 64.60 \\
\hline 98 & 35.20 & 38.40 & 65.40 \\
\hline 99 & 38.20 & 39.00 & 68.60 \\
\hline
\end{tabular}


Tabla 13

Baremos pata varones

\begin{tabular}{|c|c|c|c|}
\hline PC & Consumo por compulsión & Consumo por evasión & Escala total \\
\hline 1 & 16.00 & 16.54 & 34.00 \\
\hline 2 & 17.00 & 17.00 & 34.16 \\
\hline 3 & 17.00 & 17.00 & 36.00 \\
\hline 4 & 17.00 & 17.00 & 37.00 \\
\hline 5 & 17.00 & 17.00 & 37.00 \\
\hline 10 & 18.00 & 18.00 & 39.40 \\
\hline 15 & 18.00 & 19.00 & 41.10 \\
\hline 20 & 18.00 & 20.00 & 42.80 \\
\hline 25 & 19.00 & 20.00 & 44.50 \\
\hline 30 & 19.00 & 21.00 & 46.00 \\
\hline 35 & 20.00 & 21.00 & 46.00 \\
\hline 40 & 20.00 & 22.00 & 48.00 \\
\hline 45 & 21.00 & 23.60 & 49.00 \\
\hline 50 & 22.00 & 25.00 & 50.00 \\
\hline 55 & 22.00 & 26.00 & 51.70 \\
\hline 60 & 24.00 & 28.00 & 52.00 \\
\hline 65 & 25.00 & 29.00 & 54.00 \\
\hline 70 & 26.00 & 30.00 & 55.00 \\
\hline 75 & 28.00 & 32.00 & 56.00 \\
\hline 80 & 30.00 & 33.00 & 57.00 \\
\hline 85 & 31.00 & 35.00 & 58.00 \\
\hline 90 & 32.60 & 36.00 & 60.00 \\
\hline 95 & 35.30 & 38.00 & 63.30 \\
\hline 96 & 36.00 & 38.84 & 64.00 \\
\hline 97 & 37.76 & 39.38 & 65.00 \\
\hline 98 & 39.92 & 40.00 & 65.00 \\
\hline 99 & 41.92 & 41.38 & 73.90 \\
\hline
\end{tabular}

Las tablas 12 y 13 presentan percentiles que corresponden a los puntajes directos o brutos que se obtienen en la Escala de Consumo Patológico, según las dos dimensiones que conforman esta escala y considerando el sexo.

Las puntuaciones de 36-44 y 34-45 para mujeres $\mathrm{y}$ hombres, respectivamente, indican que existe un nivel de consumo normal, las puntuaciones de 45-51 y 46-50 indican que existe indicadores de patología en un nivel leve, conforme aumentan los puntajes de 52-55 y 51-56 se ubican en un nivel de consumo moderado. Finalmente las puntuaciones de 56-68 y 57-73 indicarían un nivel de consumo severo. 


\section{DISCUSIÓN}

Los resultados obtenidos de este estudio han permitido avalar la construcción y validación de la Escala de Consumo Patológico, con las evidencias preliminares de su confiabilidad y validez. Los resultados se determinaron a través de una muestra conformada por adolescentes del cuarto y quinto año de secundaria y nivel preuniversitario de Lima Metropolitana.

Además, se realizó un estudio piloto, donde la muestra estuvo conformada por 32 estudiantes del colegio Unión de Ñaña. Los datos obtenidos pasaron por un análisis estadístico, cuyos resultados alcanzados fueron significativos, lo que permitió que se aplicara el instrumento a una muestra más grande, siendo esta de 320 adolescentes.

Para determinar la validez del instrumento diseñado, se ejecutaron diferentes estudios, con el fin de mostrar evidencias científicas que realmente cumple con el objetivo de medir lo que se desea que mida, tal como lo declara Abad et al. (2006), definiendo la validez como el grado en que el instrumento mide el atributo que se desea medir.

La validez de contenido del instrumento fue ejecutado mediante la validez de criterio de jueces; aplicándose el coeficiente $\mathrm{V}$ de Aiken, para la obtención de indicadores de validez, según como propone Escurra (1988). De esta manera los hallazgos presentaron que los ítems alcanzan niveles adecuados de significación estadística en cuanto a claridad, sencillez y precisión de reactivos para diagnosticar el consumo patológico.

En relación a la validez de constructo, siendo que el KMO presentó un valor de 0.870 indicando la posibilidad para el uso del análisis factorial, como lo propone García y Musito (1999) y Tomás y Oliver (2004), se consideró pertinente someter a un análisis más riguroso por medio del análisis factorial. Proyectándose, como resultados, un nivel de significancia alto; posteriormente al efectuarse el análisis por componentes rotados de Varimax, para identificar las dimensiones; se obtuvo como resultado dos factores que muestran adecuadas correlaciones, los cuales se sostienen en el marco teórico propuesto, tales factores se identifican como 1) consumo por compulsión y 2) consumo por evasión. Sin embargo, la varianza explicada de la escala de consumo patológico presenta un valor de $35.124 \%$, no cumpliendo con el nivel de varianza recomendado que es de más del 40\% (Espinoza, 2008), probablemente debido a que el instrumento solo cuenta con dos dimensiones y además Espinoza (2008) recomienda establecer más de cuatro dimensiones; por lo que se invita a investigaciones futuras el incremento de las dimensiones para hallar un valor significativo en cuanto a la varianza explicada.

A diferencia de la Escala de hábitos y conductas de consumo, creada y diseñada por Ortega y Rodríguez (2003) en Colombia que, mediante el análisis factorial exploratorio, obtuvieron cinco factores, de los cuales solo tres presentaron patrones de correlaciones claramente interpretables y elevada consistencia interna. Sin embargo, al ejecutar el análisis factorial confirmatorio, presentaron resultados con un ajuste insuficiente, según los modelos basados en la investigación.

Por su parte, el cuestionario sobre opiniones y actitudes sobre la compra diseñado por Kantar Worldpanel, al realizar el análisis factorial, obtuvo 6 factores consistentes; siendo el valor de su varianza explicada superior al 62\% y su Alfa de Cronbach mayor al 0.70 (Ledesma y Gómez, 2011).

La Escala de Consumo Patológico, para la población de Lima Metropolitana, presenta un nivel alto de fiabilidad, ya que se obtuvieron indicadores de consistencia interna a nivel general, mediante el Alfa de Cronbach (0.791), de igual manera, para amabas dimensiones, obteniendo la dimensión consumo por compulsión un Alfa de Cronbach (0.844) y la dimensión de consumo por evasión un Alfa de Cronbach (0.879), superando de esta manera los valores de punto de corte de 0.70 . Similar a la Escala de Compra Patológica de 46 ítems con cuatro dimensiones que alcanzó un valor alfa superior a (0.80), lo que indica significancia en cuanto al nivel de validez (Luna, 2011).

Mientras que Cala et al. (2011) en El Bosque, Colombia, diseñaron y construyeron una prueba para medir la compra compulsiva en la población colombiana, obteniendo como resultado una prueba de 36 ítems con tres dimensiones que aún no ha sido validado por consistencia interna, por lo que los autores motivan a investigaciones futuras.

Seguidamente, se evidencia que existe un correlato entre las dimensiones consumo por compulsión, consumo por evasión y el test en general que se obtiene aplicando el análisis de ítems que sirve para visualizar si el instrumento mide el atributo para el que fue elaborado, como lo plantea Magnusson (1991), al mencionar que la relación entre un ítem y el test total puede expresarse en términos de coeficientes de 
correlación. Entonces al correlacionar los ítems con el test, por medio del Coeficiente de Correlación Producto.

Momento de Pearson (r), se recogió como resultado una significancia al $\mathrm{p}<0.05$, evidenciando validez de los factores consumo por compulsión y consumo por evasión de la escala de consumo patológico.

Posteriormente en cuanto al objeto de medición de la escala, se encontró que el modelo de entrevista semiestructurada "Minnesota de trastornos del control de los impulsos" propuesta por Black (2010), la cual evalúa las conductas y cogniciones de compra, coincide parcialmente con la Escala de Consumo Patológico que también evalúa estructuras cognitivas, las cuales determinarán una conducta consumista que se diagnosticará como patológica o normal, sin embargo, se distingue de los demás instrumentos en que, además de medir la patología, la distingue en dos aspectos: un consumo por compulsión o consumo por evasión, sostenido en un modelo cognitivo conductual.
Finalmente se puede concluir que después de haber pasado por un análisis estadístico, la Escala de Consumo Patológico presentó resultados objetivos que garantizan su validez y fiabilidad, convirtiéndose en un aporte científico, ya que en el contexto peruano no se ha encontrado una escala que mida el riesgo de la variable estudiada, motivando su uso y aplicación para la promoción y prevención de esta patología, así como su utilidad en el área clínica y educativa, como en psicoterapia.

Por último, se recomienda tomando en cuenta los resultados de la varianza explicada (35\%), eliminar o reformular los ítems con baja carga factorial en su dimensión, y así incrementar el nivel de varianza para tener una mayor validez. Del mismo modo, se insta su aplicación a muestras más grandes, tomando como base este estudio para la realización de un análisis de fiabilidad y análisis factorial confirmatorios. 


\section{REFERENCIAS BIBLIOGRÁFICAS}

Abad, F., Garrido, J., Olea, J. y Ponsoda, V. (2006). Introducción a la psicometría: teoría clásica de los test y teoría de la respuesta al item. Madrid: La Católica. Recuperado de: http://aprendeenlinea.udea.edu.co/lms/ investigacion/file.php/39/ARCHIVOS 2010/ PDF/ IntPsicometria aristidesvara_1_.pdf

Aravena, V., Herrera, V., Poblete, P. y Vera, D. (2006). Consumo patológico: compra impulsiva y compulsiva. (Tesis inédita de licenciatura). Universidad de la Frontera, Chile. Recuperado de: http://www. inpsicon.com/estudios realizados/espanol/Aravena Esp_04052007.pdf

Bauman, Z. (2007) Vida de consumo. Buenos Aires: Fondo de Cultura Económica. Recuperado de: www. Librosintinta.in

Blak, M. (2010). Compra compulsiva: una revisión. Revista de Toxicomania y Salud Mental, 28(3), 61 - 413. Recuperado de:alfama.sim.ucm.es/wwwisis2/wwwisis. exe/[in=psylink.in]/?mfn.

Cala, M., Beltrán, M., Cubillos, D. y Molano, M. (2011). Diseño de una prueba para medir compra compulsiva en población colombiana. Revista Hispanoamericana de Psicología, 11 (2), 70 - 76. Recuperado de: http://www. uelbosque.edu.co/sites/default/files/publicaciones/ revistas/cuadernos_hi panoamericanos_psicologia/ volumen11_numero2/articulo_4.pdf

Choliz, M. (2013). Tratamiento cognitivo conductual en un caso de adicción a internet y videojuegos. 13 (1), p. 125 - 141. Recuperado de: http://www.redalyc.org/ revista.oa? $\mathrm{id}=560$

Delgado, J. (2010). El síndrome de las compras: consumo y consumopatía. Revista de Salud Mental,20(8), 1115. Recuperado de: http://www.rinconpsicologia. com/2010/01/el-sindrome-de-las-comprasconsumismo-y.html

Diario Gestión. (2013). Ventas de supermercados bordearian más de 10000 millones este año. Lima, Perú martes 02 de julio. Recuperado de: http://gestion.pe/ economia/ventas-supermercados-bordearian-s-10000millones- este-ano-207012
Espinoza, J. (2008). Validación y estandarización de instrumentos. COLPARMEX, 15(9), p.4-16. Recuperado de: http://extension.upbbga.edu.co/web2/ pagina2/archivos/VYEInstrumentos.pdf

Faber, R., O'Guinn, T. y Krych, R. (1987). Consumo compulsivo. Avances en Investigación del Consumo, 4 (14), 132-135.

Faber, R., O'Guinn, T. y Krych, R. (1987). Consumo compulsivo. Avances en Investigación del Consumo, 4(14), 132-135.

Ledesma, C. y Gómez, J. (2011). Posicionamiento del minorista y motivaciones del comprador de marca del distribuidor. 3 (1), p. 50. Recuperado de: http:// www.webislam.com/articulos/31433-la_sociedad_de consumo.html

Luna, Q. (2011). Compra patológica. Revista de Psicología, 8(19), 459-469.Recuperado de: http://www.psicoactiva. com/o_i.htm /

Moreschi, G. (2006). Locos por comprar. Compra y compradores compulsivos: una patología, 4(8), 5-7 .Recuperado de: http://www.psygnos.net/biblioteca/ articulos/toc/compradorescompulsivos.htm

Ortega, G. y Rodríguez, M. (2003). Construcción de la escala de hábitos y conductas de consumo. (Tesis inédita de licenciatura). Universidad de Caldas, Colombia.

Rodríguez, M. (2001). Temas de sociología. MadridEspaña: Huerga y Fierro Editores S.L. Recuperado de: www.books.google.com.pe

Santamarta, J. (2007). La sociedad del consumo: El interior de un hipermercado. España. 3 (1), 50. Recuperado de: http://www.webislam.com/articulos/31433- la sociedad de consumo.html 